\title{
Free Play in Contemplative Ambient Intelligence
}

\author{
Douglas H. Fisher ${ }^{1}$ and Mary Lou Maher ${ }^{2}$ \\ ${ }^{1}$ Vanderbilt University, douglas.h.fisher@ Vanderbilt.Edu \\ ${ }^{2}$ University of Maryland, mlmaher@umd.edu
}

\begin{abstract}
This paper introduces free play, a meaning making activity, as a desideratum of social and contemplative ambient intelligence. A contemplative AmI is not focused on easing routine human activities, but through free play and other mechanisms, will encourage humans to engage with each other and the AmI on thinking about and acting on societal issues over long time scales. These ideas are illustrated by the design of an interactive, intelligent art installation about adaptation to climate change. This approach to AmI extends the connotations of AmI along social, spatial, and temporal dimensions.
\end{abstract}

Keywords: Free play, collective intelligence, social intelligence.

\section{Characteristics and Dimensions of Ambient Intelligence}

"Digital environments that are sensitive and responsive to the presence of people" [1] is a Phillips' definition of ambient intelligence (AmI), which they further characterize as embedded, context aware, personalized, adaptive and anticipatory [1]. Moreover, many treatments of AmI further connote that embedding be 'invisible'; that 'personalization' be relative to an individual, rather than say a group; that 'anticipatory' actions be 'unobtrusive', seamlessly complementing human users.

Consider that 'calmness' and 'invisibility' are often forwarded as desiderata of pervasive technology generally [2], but the claim for invisibility is often limited to the technology's use phase. For example, sensors embedded in clothes may be invisible during the clothing's use, but this technology may be anything but invisible or calm if at the end of the clothing's life it is thrown into a garbage heap. To be truly invisible, the technology must be hidden and calm over its full lifetime, and over the lifetimes of many generations of the technology; otherwise it may in fact be harsh technology.

We seek to expand connotations of AmI in three spaces. Along social dimensions, AmI can be highly visible, proactively engaging participants in a larger community on critical social issues. Along spatial dimensions AmI is typically associated with appliances, rooms, homes, office buildings, and cities [2], but AmI can also extend to virtual worlds. Along temporal dimensions, latency of response can vary from instantaneous to the result of lengthy contemplation.

In most cases AmI is designed for an existing corpus of activities. However, a technology, even an 'invisible' one, can't help but to change human behavior. For example, environmentally 'smart buildings' may contribute to a glut of environmentally stupid people [3]; this is but one example of 'higher-order effects' on human behavior of pervasive computing [4], particularly when we look to larger social scales beyond the individual. Even simple changes to AmI designs may have 
important consequences on human participants; for example, an AmI that turns lights out after a period of no motion has possibly removed any concern with energy efficiency from the consciousness of the transitory occupant, whereas a more intelligent, context aware, and provocative AmI that turns lights out as (not after) the last person exits may actually raise human consciousness. In general, we can design AmI to be a proactive part of the social system, and design the activities with AmI in mind, all of which is consistent with activity theory and interaction design [5].

AmI that is 'calm' on small time and social scales may be negatively disruptive when viewed on larger scales. Inversely, it may be that AmI that is benevolent at large scales may be best achieved, at least in some cases, by technology that is visible and questioning, even provocative, at smaller scales.

In this landscape paper we introduce contemplative AmI, and given space constraints, we focus on but one design principle for social and contemplative AmI, the meaning making activity of free play. Section 2 introduces the free play paradigm and surveys some of its literature, with links to human-computer interaction (HCI) and experience design as appropriate. Section 3 illustrates free play in the context of an interactive art installation that is under development; this will be an AmI that engages in a kind of contemplative discourse with humans.

\section{Free Play and Meaning Making}

"Free Play is the creative activity of spontaneous free improvisation, by children, by artists, and people of all kinds." [6] The idea of free play has been described in contrast to competitive play: free play engages people to participate in playful activities that focus on meaning making and creativity, and competitive play engages people to participate in playful activities in which winning is the goal [7][8]. Free play has also been described as open-ended exploration, and an infinite game, played for the purpose of continuing the game [9]. Web 'surfing' is a good example of free play. While free play has open-ended aspects, this often includes goal driven activity in which the user selects and then pursues goals intermittent with exploration.

Gaver [10] has explored ludic engagement and ludic design of HCI-based interactive experiences, which favors design for pleasure, to include open-ended exploration, over function. In both HCI and AmI the emphasis has been to design for function, productivity and efficiency. Moving from function to pleasure has some precedents: environments and objects/toys can be designed specifically to encourage free play. For example, toys such as LEGO® provide objects that children can put together to create new objects or games. Virtual environments support free play by allowing people to build their own objects; the virtual world itself, appropriately instrumented, could be regarded as an AmI. In all of these contexts, goal-driven behavior frequently emerges, such as building an envisioned LEGO structure.

In contrast to toys and virtual worlds, we are beginning to see free play that invites participation, for example, in open-ended interactive art. The artist's intent behind the work is often to attract and initiate exploration by participants, that is, to invite free play, rather than to be invisible. Interactive art installations are often proactive AmIs, though they vary in the social, spatial and temporal extents that they engage humans. 
Goldberg [11] has created numerous robotic and internet-based art installations to provoke interaction and reaction. Goldberg's Robotic Tele-garden engaged thousands of people, many of them for years, in watering and seeding a common garden through tele-robotic controls available on a web page; on short time scales, individual actions like watering a plant are goal driven, but at large temporal scales the garden design 'dances' and the collective of gardeners can be viewed as engaged in free play. Merrick, Maher \& Saunders [12] developed a curious information display that changed in response to its ability to attract the attention of the people in the room. Morrison, Miller \& Viller [13] consider interactive art installations that provoke reflection through gesture.

HCI evaluation of interactive art is being explored in contrast to evaluating HCI for utility and efficiency [7]. Costello and Edmonds [14] focus on interactive art that stimulates playful behavior to achieve a deep level of engagement. They identify thirteen stages in a pleasure framework for interactive art. They used this framework to evaluate three installations, and the significant difference in experience occurred in the installation that has one open-ended play level. The participants reported that once they had "figured it out" they would move on. In contrast, we also want AmI to support infinite play, which engage people in exploration for long periods of time.

AmI that is designed to support, encourage and participate in free play can engage individual human thought and lead towards a collective intelligence with respect to social issues. Importantly, free play in AmI doesn't happen in a vacuum - the environment necessarily constrains the boundaries of exploration and play, facilitating constructivist human learning [15]. Finding the sweet spot between over and under constraint of exploration is a design challenge [10].

\section{Encouraging Free Play on Ideas about Climate Change}

To illustrate an AmI designed to provoke and affect our thinking, we describe a hypothetical knowledge-based, interactive art installation that promotes public dialog on climate change. Figure 1 shows the system (under development) in three layers: an artistic rendering (in front), a knowledge base (i.e., a concept or topic map), and an incoming content layer. Rather than being invisible, a large interactive display of the virtual world will be in a public location, giving both physical and virtual spatial extent to the AmI. When an observer approaches motion sensors will trigger activity, such as a meandering virtual tour. Beyond passive observation, the installation will encourage/support passersby' participation in several ways:

1. To explore the artistic rendering of climate change concepts by touching the screen and by moving an exhibit avatar through the virtual village; such exploration is one aspect of free play in this context.

2. To contribute commentary through online text and image messaging, which can be displayed in real-time; as well as substantive documents (e.g., class projects) offline to the installation's servers by email or Web interface (see below). These can all be the basis for asynchronous community dialogue. 
3. User history informs AmI reactions, such as teleporting to a new location based on these histories with the exhibit; AmI reactions can be provocative, teleporting users to counterpoints of their own opinion, for example.

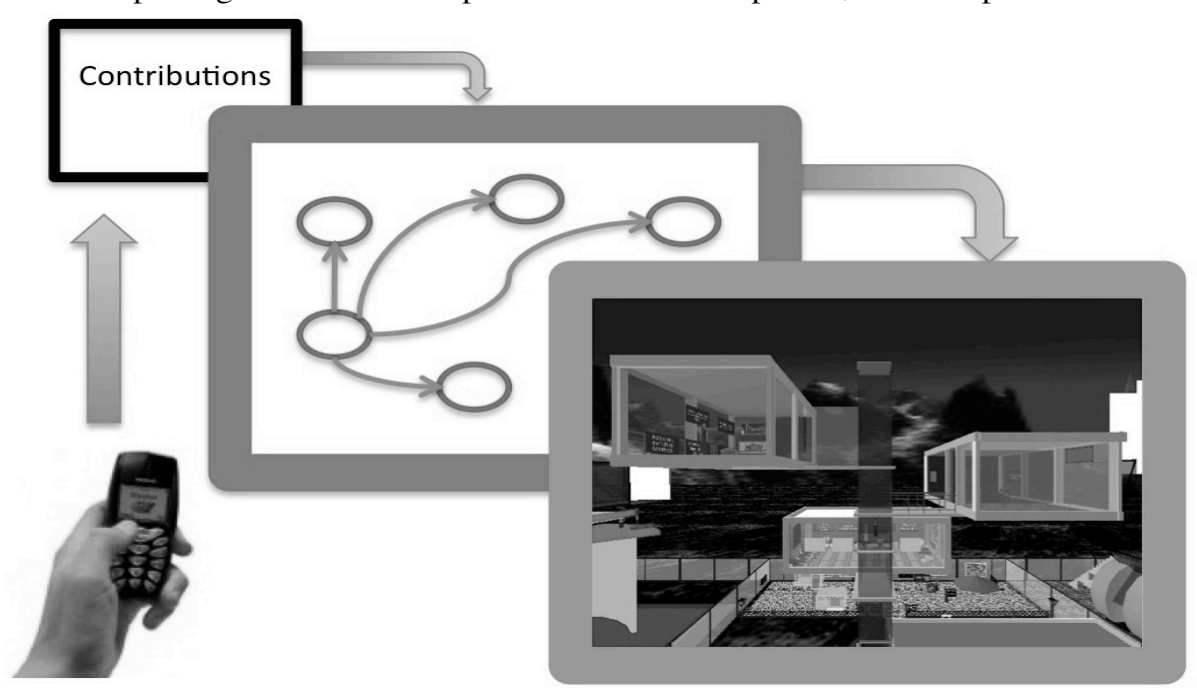

Figure 1: An overview of an interactive art installation for engaging the public in creatively solving social issues (virtual world image is author and student created content in ActiveWorlds).

Machine learning methods of topic modeling and clustering (e.g., [16, 17]) will continually process commentary and substantive document contributions, deciding where they conceptually fit into the concept/topic map of the installation's middle layer, and changing those knowledge structures as a result. As the concept map evolves, so will the virtual village that organizes its content, since concepts will map onto virtual physical locations like different thematic rooms in a museum.

The virtual village and underlying concept map provide structure for exploring content and contributions of others. Individuals can "play" with ideas by touching, selecting, contributing and commenting. We intend "play" to extend well beyond fantasy and entertainment; we endeavor to create an environment for long-term socially responsive discourse. The proposed installation is similar in form and intent to Helsinki's City Wall [18], but there is no artificial intelligence in CityWall.

Context awareness is, in part, real-time, through motion sensors, touch and instant messaging identifiers of users. But context awareness also occurs in the realm of ideas (i.e., where do contributions fit relative to the concept map), and through these "thick" inputs, an awareness of where authors reside in this space. Response in this latter case takes the form of placing an author's work in a larger conceptual context, to be found later by the same authors and others; this response latency is not immediate, but based on machine-intelligent deliberation, all consistent with a desire for contemplation on issues of long-term planet sustainability. We anticipate that point/counterpoint works, as well as those exhibiting shared opinions, will be displayed side-by-side by the AI in the AmI, thereby provoking further human discourse over long time intervals. 


\section{Conclusion}

AmI can be extended beyond supporting actions, to reinforce peoples' predispositions, to proactive environments in which free play and machine intelligence raise awareness in people and heighten their thinking. We suggest an extension of the original ambit of AmI, to include a highly visible social and collective intelligence, including machine learning that encourages thought and action on important societal issues. Generally, our prescription for contemplative AmI begs a more complete description as well - to characterize and distinguish current and future AmI along social, spatial and temporal dimensions.

\section{References}

1. Philips, www.research.philips.com/technologies/syst softw/ami/background.html.

2. Cook D.J, Augusto J.C, Jakkula V. R.: Ambient intelligence: Technologies, applications, and opportunities, Pervasive and Mobile Computing, 5(4), pp 277-298 (2009)

3. Peterson, J., Shunturov, V., Janda, K., Platt, G. and Weinberger, K.: International Journal of Sustainability in Higher Education, 8(1), pp 16-33 (2007)

4. Köhler, A.; Erdmann, L.: Expected Environmental Impacts of Pervasive Computing. Human and Ecological Risk Assessment, No 10, pp 831-852 (2004)

5. Kaptelinin, V., Nardi, B.: Acting with Technology: Activity Theory and Interaction Design. Cambridge: MIT Press (2006)

6. Wikipedia, http://en.wikipedia.org/wiki/Free Play: Improvisation in Life and Art

7. Morrison, A., Mitchell, P., and Brereton, M.,: The lens of ludic engagement: Evaluating participation in interactive art installations. In Proceedings of the 15th International Conference on Multimedia, ACM Press, Augsburg, Germany, 509-512 (2007)

8. Flemmert Jensen, A.:Time for Playful Learning? - A cross-cultural study of parental values and attitudes towards children's time for play, Lego learning institute, DK (2002)

9. Carse, J.P.: Finite and Infinite Games: A Vision of Life as Play and Possibility. Ballantine Books, New York (1987)

10. Gaver, B.:Designing for Homo Ludens. 13 Magazine No 12, June (2002)

11. Goldberg, http://goldberg.berkeley.edu/art/index.html.

12. Merrick, K., Maher, M-L., Saunders, R.:Achieving Adaptable Behaviour in Intelligent Rooms using Curious Supervised Learning Agents, CAADRIA 2008, Beyond Computer Aided Design, Chiang Mai, Thailand, pp 185-192 (2008)

13. Morrison, A., Mitchell, P., and Viller, S.: Evoking Gesture in Interactive Art, HCC '08 Proceedings of the 3rd ACM International Workshop on Human-centered Computing, ACM, pp 11-18 (2008)

14. Costello, B. and Edmonds, E.: A study in play, pleasure and interaction design, DPPI '07 Proceedings of the 2007 Designing Pleasurable Products and Interfaces, (2007)

15. Brewer, J., Burke, M., Fenty, L., Patton, D., Post, J., Simpson, H. Learning Methodology Reference Document, NAVSEA Performance Monitoring, Training and Assessment Program Office (PMS430) http://www.icte.org/T98 Library/LMREFDOC.PDF (1999).

16. Hoffman, M., Blei, D. and Bach, F.: Online Learning for Latent Dirichlet Allocation, in Proceedings of Neural Information Processing Systems, (2010).

17. Fisher, D. : Iterative Optimization and Simplification of Hierarchical Clusterings, Journal of Artificial Intelligence Research, 4:147-179, (1996).

18. CityWall, citywall.org 